\title{
Increase in visual motor coordination after applying mCIMT in infantile hemiplegia. Report of a case
}

\author{
Palomo C Rocío* and García-Muro San José \\ Department of Physiotherapy, CEU-San Pablo University of Madrid, Spain
}

\begin{abstract}
Introduction: children with hemiplegia have a deficit in the motor behaviour that interferes with grip strength and the visuomotor coordination of the upper limb during the reach an object. The non-use of the paretic upper limb because of that a lack of integration into the body schema, produces a weakness in manipulative dexterity.
\end{abstract}

Aim: To assess the increase in visual motor coordination and grip strength of the affected upper limb in a girl with hemiplegic applying a modified constraint-induced movement therapy (mCIMT) protocol.

Methods: Clinical case and intervention: A protocol was applied for five weeks, two hours per day in a girl was 5-year-old with infantile hemiplegia, a level II in the manual ability classification system, MACS. Four measurements are used in time. Visual motor coordination is assessed by catching an object in motion. The grasp is also assessed in static and dynamic.

Conclusion: An increase in visual motor coordination and grip strength of the paretic limb could possibly be obtained in the child when applying constraint-induced movement therapy into the usual environment.

\section{Introduction}

Infantile cerebral palsy (ICP) is defined as a series of permanent disorders that affect the child's motor and postural development. Functional limitations occur in their motor activity, triggered by the brain injury produced during the maturation of the central nervous system of the foetus or infant. ICP is characterized as a non-progressive encephalopathy. It is a chronic sensorimotor disorder that leads to the alteration of sensitivity, perception, cognition, communication and behaviour, in addition causing changes in posture, muscle tone and production of inappropriate movements. These alterations are usually caused by secondary problems of cerebral palsy, such as epilepsy and musculoskeletal disorders, which is the same as those of the brain. The prevalence of ICP in developed countries is 2 to 2.5 cases per 1,000 live births [1].

Children with hemiplegia (subtype of ICP) are characterized by deficits in motor behaviour accompanied by stereotyped movements and poor postural adjustments that interfere with the balance, reaction time and speed of reaching an object. Therefore, they need a movement time four times higher than a child without neurological problems. The affected hand is accompanied by a deficit in proprioception and tactile perception, which hinders the fine motor skills, in general of the fingers, in addition to the strength exerted. There is no balance between proprioceptive and visual spatial information, thus altering the visual-motor coordination of the affected limb. The movements are slower and with awkwardness in the coordination, longer during the execution, with associated movements and in a mirror. The presence of mirror movements hinders the coordination of the affected upper limb. In children with hemiplegia they are increased and prolonged in time by the lack of selective control and presence of spasticity altering the bimanual coordination, which leads to a decrease in the use of the affected hand that usually interferes with the manual ability of the healthy upper limb [2].

From the first stage of childhood, children with hemiplegia, even those with the least affectation, tend to use their healthy hand as dominant in all tasks. Therefore, they learn not to use their affected arm, which is known as "Disregard of development" [3] This "nonuse" of their affected upper limb produces an increase in muscle tone in this body segment, poor motor control, decreased muscle range of active and passive movement, generalized weakness and delay of musculoskeletal maturation. This deterioration of non-use is caused by a neural dysfunction during brain injury [4].

Constraint Induced movement therapy (CIMT) is an intervention technique to improve the use and functionality of the affected upper limb in patients with brain injury. It was described by Taub et al. [5] in 1980 and is based on "learning not to use the affected upper extremity".

The therapy began to be applied in primates later transferring to adults and consisted in the restriction of the non-paretic upper limb through a means of containment (sling, splint...) thus promoting the use of the affected limb in the activities of daily life. The restraint was maintained during $90 \%$ of the waking hours. 6 consecutive hours of intensive treatment were performed in a clinic with exercises of

*Correspondence to: Palomo Carrión Rocío, Calle Lope de Vega, 8. Torrijos, Toledo, 45500, Spain, E-mail: rociopalomotoledo@gmail.com

Key words: infantile hemiplegia, neuroplasticity, physiotherapy specialty, exercise therapy, strength hand, movement disorder

Received: November 27, 2018; Accepted: December 14, 2018; Published: December 18, 2018 
manipulative skill during a period of 10 to 12 days. The tasks integrated the repetition of the motor action with a variety of exercises. This repetition allowed the acquisition of motor learning in the paretic hand $[5,6]$.

Different studies [7-9] confirmed that the merits acquired during the rehabilitation period were maintained between six months and one year after the intervention. After the application of Constraint Induced movement therapy following the use of the forgotten part, the stimulation of areas of the brain that had previously been inactivated by the use of the forgotten part was observed by means of magnetic resonance. An adaptation of the protocol is needed because most of the children did not complete the treatment because they did not exceed the full time of restriction, known as modified Constraint Induced movement therapy (mCIMT) [10,11].

Aim: "To assess the increase in visual motor and grip strength of the affected upper limb in a girl with right hemiplegia of 5 years old after applying modified Constraint Induced movement therapy"

\section{Methods}

\section{Clinical case definition}

The study is conducted on a 5-year-old girl who suffered brain damage during birth (perinatal injury) due to fetal distress.

She was diagnosed with right spastic hemiplegia at 15 months of age when she attended day care, since inactivity was detected in the right upper extremity when performing bimanual tasks. Magnetic resonance diagnosis was confirmed, showing a cortico-subcortical lesion adjacent to the left central groove with gliosis foci and malacia. In addition, the ectatic Trolard vein is observed and, however, the lateral ventricles and third ventricle appear in a normal situation, size and morphology.

The girl has motor difficulties that show changes in the gait, of static and dynamic balance, obtaining an assessment on the scale of the case of Berg [12] independence (total score $=43$ ) to be able to walk without limitations and not require a wheelchair. After an assessment of their motor skills, the girl is placed in level I of gross motor classification System in children with cerebral palsy, GMFCS [13].

There is a limitation of the manual functionality of the right upper extremity being found in a level II within the classification system of manual skill in children with cerebral palsy, MACS [14]. Its most pronounced deficit is manifested in fine motor skills, in the execution of manual motor skills with dexterity, and in the visual-motor coordination of the hand, since it requires a longer time to perform any activity.

In order to be able to apply the therapy, certain criteria of inclusion are considered [15].

- Lack of activity of the affected upper limb.

- Overcoming the $10^{\circ}$ extension in the metacarpophalangeal and interphalangeal joints.

- Having more than $20^{\circ}$ of extension of the wrist of the affected upper extremity.

- Adequate cognitive development to understand the verbal orders given for the execution of tasks.

- Cooperation in the execution of them.

The following were established as exclusion criteria:

- Have associated pathologies
- Have uncontrolled epilepsy

- Severe spasticity that prevents functional movement of the affected upper limb.

\section{Method of intervention}

The study is carried out in a period of 5 weeks of treatment, using a time of restriction of the healthy upper extremity of 2 hours per day (one hour in the morning and one hour in the afternoon) from Monday to Friday. An informed consent is given to the family for the execution of the therapy. The intervention protocol and informed consent were approved by the ethics committee of the San Pablo CEU University of Madrid.

The restriction used, is a partial, which consists of keeping the healthy hand closed and the thumb inside the fist by means of a transparent film that reaches the wrist joint, and that mimics the restriction of a glove [16] but it is more comfortable for the development of activities. Handling is prevented with the unaffected hand leaving the wrist and elbow joint free so that the girl can react to an external disturbance.

\section{Primary measures}

The visual motor coordination is divided in three variables, the reaction time, the total movement time, both expressed in seconds (Figure 1) measured through a chronometer and the reach of the affected upper limb determined by stopping a moving object. (registered in centimeters) that slides on a circuit with a wooden ramp and an adjustable slope at $10^{\circ}$ or $15^{\circ}$. The reach of the girl will be greater the smaller the distance at which the car in motion is stopped. For the measurement of visual-motor coordination (eye-hand) and the manual grip on dynamic of the paretic hand, the circuit used in the study of Mak adapted to our needs is used [17]. The circuit consists of a sloping wooden board (ramp), located on a wooden base and with a gradient of the ramp adjustable to $10^{\circ}$ and $15^{\circ}$. At the top end of the board is a toy car weighing 80 grams held by an electromagnet. The car has a builtin manual dynamometer that marks the dynamic grip strength of the affected hand when the girl takes the car while traveling on the wooden ramp. The girl remains seated in a chair homolateral to the affected upper limb in situation to the table where the board is located. She must press a switch, placed at the bottom end of the ramp, as quickly as possible when She receives the verbal command of the evaluator "already" for the car to go down a lane located on the ramp. The lane is numbered from 0 to 77 centimetres. The car starts from an initial position of $0 \mathrm{~cm}$ and will go down at a speed of $0.42 \mathrm{~m} / \mathrm{s}$ for the slope of $10^{\circ}$ and to $0.65 \mathrm{~m} / \mathrm{s}$ for the slope of $15^{\circ}$ (Figure 1).

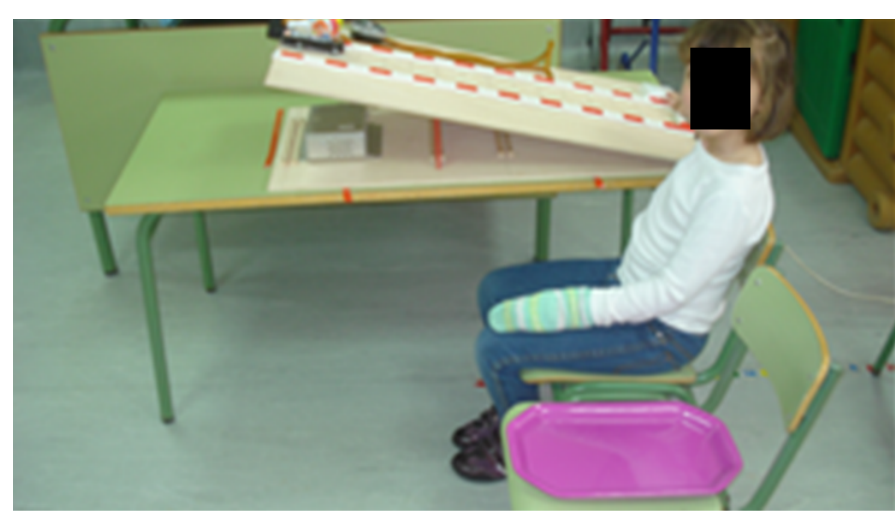

Figure 1. Visual-motor coordination test 


\section{Secondary measures}

The hand grip strength dynamic is registered with a manual dynamometer, using the same scale units that the grip static dynamometer $(0-150 \mathrm{psi})$. It is included in a moving object that moves through a $10^{\circ}$ or $15^{\circ}$ slope as adjusted [18].

\section{Estadistic analysis}

In the variable of visual-motor coordination, a statistical analysis was carried out using the statistical program PASW 18. A repeatedmeasures ANOVA is used to see its statistical significance throughout the measurements ( $95 \%$ confidence interval).

\section{Results}

\section{Primary outcomes}

We take the means of the values obtained in the successful trials of each slope.

In the circuit of slope $10^{\circ}$ the five successful tests are completed, unlike the $15^{\circ}$ slope where in the first measurement 4 are completed, $80 \%$, increasing totally to the rest of the measurements.

The variables studied corresponding to the reaction time, total Movement time and distance at which the car stops at the affected upper extremity, without distinction of slopes, suffer a reduction in the values obtained from each of the assessments made. Changes are observed in each of the measurements, resulting in the most important increase in the difference of values from the first to the fourth measurement with a $p$ value $<0,05$. The reaction time values on both slopes decrease in parallel, because of that the slope is not an influential factor in the Results $(p>0.05)$. In contrast, the values obtained in the total movement time suffer a greater reduction in the $10^{\circ}$ slope since they start from a higher initial value than in the $15^{\circ}$ slope (Figure 2 ). The difference in the value recorded between the first and the fourth measurement for the study of the distance at which the car stops (the affected upper limb's reach of the girl) is greater on the $15^{\circ}$ slope than on the lower slope inclination. Consequence of the influence of the slope on the results of the variables studied (total movement time and range of the affected upper limb). In general, the results obtained have a more remarkable improvement in the $10^{\circ}$ slope (Tables 1 and 2).

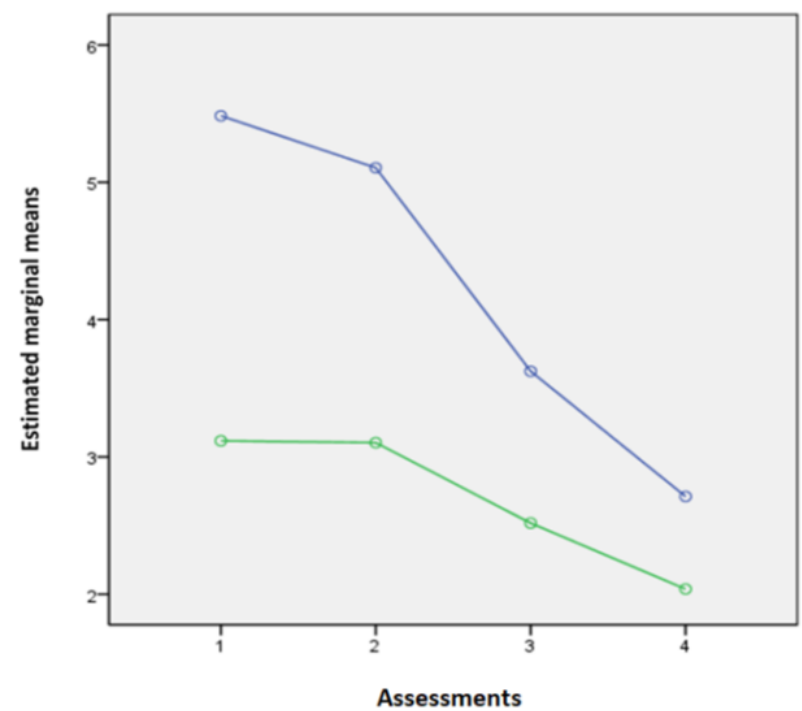

Figure 2. Progress for total movement time of the task, in the slope $10^{\circ}$ and $15^{\circ}$
Table 1. Recording the results depending on the slope used for visual- motor coordination

\begin{tabular}{|c|c|c|c|c|}
\hline Slope- $10^{\circ}$ & Assessment & Means & $\begin{array}{c}\text { Typical } \\
\text { deviation }\end{array}$ & $\mathbf{N}$ \\
\hline \multirow[t]{5}{*}{ Reaction time } & 1 & 2,4080 & 49251 & 5 \\
\hline & 2 & 1,9600 & 50384 & 5 \\
\hline & 3 & 1,7280 & 45680 & 5 \\
\hline & 4 & 1,5680 & 41288 & 5 \\
\hline & Total & 1,9160 & 53825 & 20 \\
\hline \multirow[t]{5}{*}{$\begin{array}{l}\text { Total movement } \\
\text { time of the task }\end{array}$} & 1 & 5,48 & 412 & 5 \\
\hline & 2 & 5,11 & 833 & 5 \\
\hline & 3 & 3,62 & 1,472 & 5 \\
\hline & 4 & 2,71 & 244 & 5 \\
\hline & Total & 4,23 & 1,403 & 20 \\
\hline \multirow[t]{5}{*}{ Reach/distance } & 1 & 54,00 & 2,345 & 5 \\
\hline & 2 & 51,80 & 2,775 & 5 \\
\hline & 3 & 50,60 & 2,408 & 5 \\
\hline & 4 & 50,00 & 2,449 & 5 \\
\hline & Total & 51,60 & 2,780 & 20 \\
\hline Slope- $1^{\circ}$ & Assessment & Means & $\begin{array}{c}\text { Typical } \\
\text { deviation }\end{array}$ & $\mathbf{N}$ \\
\hline \multirow[t]{5}{*}{ Reaction time } & 1 & 2,2175 & 29182 & 4 \\
\hline & 2 & 1,9960 & 18515 & 5 \\
\hline & 3 & 1,9160 & 17841 & 5 \\
\hline & 4 & 1,3920 & 12677 & 5 \\
\hline & Total & 1,8626 & 35735 & 19 \\
\hline \multirow[t]{5}{*}{$\begin{array}{l}\text { Total movement } \\
\text { time of the task }\end{array}$} & 1 & 3,12 & 500 & 4 \\
\hline & 2 & 3,10 & 222 & 5 \\
\hline & 3 & 2,52 & 310 & 5 \\
\hline & 4 & 2,04 & 255 & 5 \\
\hline & Total & 2,67 & 550 & 19 \\
\hline \multirow[t]{5}{*}{ Reach/distance } & 1 & 57,00 & 1,633 & 4 \\
\hline & 2 & 55,80 & 837 & 5 \\
\hline & 3 & 55,40 & 1,342 & 5 \\
\hline & 4 & 51,40 & 894 & 5 \\
\hline & Total & 54,79 & 2,417 & 19 \\
\hline
\end{tabular}

$N$ : number of trials, maximum of 5 trials

Table 2. The 95\% confidence interval is applied to obtain the statistical values of the variables

\begin{tabular}{|c|c|c|}
\hline Variable & factor & Sig. \\
\hline Reaction time & slope & 0,762 \\
\hline $\begin{array}{c}\text { Total movement time of } \\
\text { the task }\end{array}$ & Assessment & $0,000^{*}$ \\
\hline & Slope & $0,000^{*}$ \\
\hline Reach/distance & Assessment & $0,000^{*}$ \\
\hline & Slope & $0,000^{*}$ \\
\hline
\end{tabular}

Sig.(significance), statistically significant difference when $p$ value $<0.05$

\section{Secondary outcomes}

The dynamic grip remains stable throughout the measurements, although it shows an increase of 1 psi in the last measurement, being the same for both slopes.

The static grip starts from a value greater than the dynamic grip and suffers an appreciable increase from the third measurement, obtaining the maximum value in the fourth measurement with an increase of 2 psi from the first measurement taken. 


\section{Discussion}

The deterioration of the functionality of the hand causes a weakness present in the execution of activities of daily life in infantile hemiplegia. There is an alteration compared to the healthy upper limb that manifests itself in the general slowness of movement, discontinuous movements, variability in the trajectory of the hand with trunk offsets and presence of inadequate coordination in the grip strength of the hand [19].

It is necessary to emphasize the acquisition of a more stable and precise grip due to an increase of 2 psi of force in the static manual grip from the first measurement to the last one. Improved stability and grip strength allow for greater manual functionality when picking up objects of different size and weight and maintaining them during the execution of selective and precision activities such as throwing a small ball at a target, keeping the fork stable with the affected hand and take the food to the mouth during the feeding phase. Most children with unilateral brain injuries do not develop an adequate grip strength in the coordination of a unimanual activity carried out by the affected upper limb. A pathological pattern or an immature state of grip for their age is produced, giving rise to an inadequate synergy of the coordination strength that is related to the deterioration of the manipulative dexterity of the affected hand, depending on the level of injury [20].

The mCIMT, like the bimanual occupational therapy, has been used to optimize the functionality in spastic hands after injection with botulinum toxin type A. The comparison of both interventions in the study by Hoare et al. [21] shows a greater efficacy of the mCIMT with respect to the bimanual occupational therapy, since the mCIMT facilitated motor learning of the upper limb affected by the practice of skills and the experience of the use of the hand through mass practice. However, it was not possible to guide the learning of bimanual strategies to achieve performance in the task, since children used in most trials the dominant hand to grasp the object before thinking about the role of the affected hand [21].

Visual-motor coordination of the affected upper limb in the case presented in the study underwent a favourable evolution in the course of the intervention by means of modified Constraint Induced movement therapy. Its improvement is appreciated by obtaining a reduction of the values in the reaction time, total movement time and greater active reach of the affected upper limb when stopping the car in motion of the successful tests.

A statistically significant difference is obtained with a $p$ value $<0.01$ in the reaction time dependent on the valuation and without differences between the $10^{\circ}$ and $15^{\circ}$ slopes from the first assessment to the fourth. This suggests that the girl was able to adapt her movement to an increase in the speed of the car (with a $15^{\circ}$ slope), possibly because the task was simple and did not require a thinking strategy at different speeds.

In contrast to the reaction time, the slope of $10^{\circ}$ and $15^{\circ}$ is influential in the evaluation of the total movement time and distance at which the car is stopped with a statistically significant difference with a $p$ value $<0.01$. Better results are obtained in the total movement time for the execution of the total of the task in the $10^{\circ}$ slope, there being differences between the evaluations $(p<0.01)$ than in the $15^{\circ}$ slope $(p>0.05)$ where no there are differences between the evaluations, since when the task becomes complicated, the girl needs to modulate the trajectory of her movement to the increase of the speed at which the car drops on the $15^{\circ}$ slope to complete the task.

The manual grip strength when taking the car in motion through the affected upper limb on both the $10^{\circ}$ and the $15^{\circ}$ slope suffers the same increase of $1 \mathrm{psi}$ at the end of the intervention. The increase in slope, as well as the speed at which the car goes down does not influence the increase of hand strength in dynamic. However, when there is no speed of movement of the object; the increase in manual grip strength is greater in static than in dynamic.

If we compare the results obtained in the study with an investigation carried out in children with problems in the development of the coordination by Mak [17]. we appreciate a similarity in the influence of the slope. The results recorded when executing the moving task with a different inclination show an influence of the slope in the total movement time, but it is not relevant in the reaction time, as in the present investigation. The difference is in the grip made when picking up the car in motion that varies depending on the slope (being the peak of strength on the slope of $15^{\circ}$ ), while in the current study there are no changes in the values on the different slopes. Another possibility to consider is the difficulty that children with hemiplegia have of adjusting the correct strength when catching an object due to an inadequate synergy of the affected upper limb and an immaturity in the hand grip, which can make us think that the girl does not have enough capacity to modulate the grasp when catching an object at different speeds, since there are no changes between a slope of $10^{\circ}$ and $15^{\circ}$, since at higher speeds the girl should have made a greater force in the grip to stop the object.

\section{Study limitations}

Being an uncontrolled trial with a single case and absence of a control group, it can-not be guaranteed that the response observed (changes produced throughout the intervention with respect to the baseline situation) is exclusively due to the mCIMT protocol [22].

For future research it would be necessary to expand the sample, study the quality of movement and spontaneous use of the affected upper limb applying the same protocol and introducing a control group.

\section{Conclusion}

An increase in visual motor coordination and grip strength of the paretic limb could possibly be obtained in the child when applying constraint-induced movement therapy into the usual environment.

\section{Acknowledgments}

Acknowledge the involvement of girl's family in the study and the girl herself great interest and desire for improvement.

\section{References}

1. Monge E, Molina F, Alguacil IM, Cano R, De Mauro A, et al. (2012) Use of virtual reality systems as proprioception method in cerebral palsy: clinical practice guideline. Neurologia 318: 1-10. [Crossref]

2. Camacho-Salas A (2008) Infantile cerebral palsy: the importance of population registers. Rev Neurol 47: 15-20. [Crossref]

3. Deluca SC, Echols K, Law CR, Ramey SL (2006) Intensive pediatric constraintinduced therapy for children with cerebral palsy: randomized, controlled, crossover trial. J Child Neurol 21: 931-938. [Crossref]

4. Liepert J, Bauder H, Wolfgang HR, Miltner WH, Taub E, et al. (2000) Treatmentinduced cortical reorganization after stroke in humans. Stroke 31: 1210-1216. [Crossref]

5. Taub E, Harger M, Grier HC, Hodos W (1980) Some anatomical observations following chronic dorsal rhizotomy in monkeys. Neurosci 5: 389-401. [Crossref]

6. Taub E, Miller NE, Novack TA, Cook EW 3rd, Fleming WC, et al. (1993) Technique to improve chronic motor deficit after stroke. Arch Phys Med Rehabil 74: 347-354. [Crossref] 
7. Naylor CE, Bower E (2005) Modified constraint-induced movement therapy for Young children with hemiplegic cerebral palsy: a pilot study. Dev Med Child Neurol 47: 365369. [Crossref]

8. Charles JR, Wolf SL, Schneider JA, Gordon AM (2006) Efficacy of a child-friendly form of constraint-induced movement therapy in hemiplegic cerebral palsy: a randomized control trial. Dev Med Child Neurol 48: 635-642. [Crossref]

9. Sakzewski L, Provan K, Ziviani J, Boyd RN (2015) Comparison of dosage of intensive upper limb therapy for children with unilateral cerebral palsy: how big should the therapy pill be? Res Dev Disabil 37: 9-16. [Crossref]

10. Wallen M, Ziviani J, Herbert R, Evans R, Novak I (2008) Modified constraintinduced therapy for children with hemiplegic cerebral palsy: a feasibility study. Dev Neurorehabil 11: 124-133. [Crossref]

11. DeLuca SC, Case-Smith J, Stevenson R, Ramey SL (2012) Constraint-induced movement therapy (CIMT) for young children with cerebral palsy: effects of therapeutic dosage. J Pediatr Rehabil Med 5:133-142. [Crossref]

12. Kembhavi G, Darrah J, Magill J, Loomis J (2002) Using the berg balance scale to distinguish balance abilities in children with cerebral palsy. Pediatr Phys Ther 14: 9299. [Crossref]

13. Gray L, Ng H, Bartlett D (2010) The gross motor function classification system: an update on impact and clinical utility. Pediatr Phys Ther 22: 315-320. [Crossref]

14. Eliasson AC, Krumlinde L, Rösblad B, Beckung E, Arner M, et al. (2006) The Manual Ability Classification System (MACS) for children with cerebral palsy: scale development and evidence of validity and reliability. Developmental Medicine and Child Neurology 48: 549-554. [Crossref]
15. Ramachandran S, Thakur P (2011) Upper extremity constraint-induced movement therapy in infantile hemiplegia. J Pediatr Neurosci 6: 29-31. [Crossref]

16. Eliasson AC, Krumlinde L, Shaw K, Wang C (2005) Effects of constraint-induced movement therapy in young children with hemiplegic cerebral palsy: an adapted model. Dev Med Child Neurol 47: 266-275. [Crossref]

17. Mak MK (2010) Reaching and grasping a moving target is impaired in children with developmental coordination disorder. Pediatr Phys Ther 22: 384-391. [Crossref]

18. Newman DG, Pearn J, Barnes A, Young CM, Kehoe M, et al. (1984) Norms for hand grip strength. Arch Dis Child 59: 453-459. [Crossref]

19. Steenbergen B, Charles J, Gordon AM (2008) Fingertip force control during bimanual object lifting in hemiplegic cerebral palsy. Exp Brain Res 186: 191-201. [Crossref]

20. Kim D (2016) The effects of hand strength on upper extremity function and activities of daily living in stroke patients, with a focus on right hemiplegia. J Phys Ther Sci 28: 2565-2567. [Crossref]

21. Hoare BJ, Im C, Rawicki HB, Carey L (2010) Modified constraint-induced movemen therapy or bimanual occupational therapy following injection of Botulinum toxin-A to improve bimanual performance in young children with hemiplegic cerebral palsy: a randomised controlled trial. BMC Neurol 10: 58. [Crossref]

22. Argimon JM, Jiménez J. Methods of clinical and epidemiological research. Elsevier Third edition.

Copyright: ( 2018 Rocío PC. This is an open-access article distributed under the terms of the Creative Commons Attribution License, which permits unrestricted use, distribution, and reproduction in any medium, provided the original author and source are credited. 